QUARTERLY OF APPLIED MATHEMATICS

VOLUME LXVII, NUMBER 3

SEPTEMBER 2009, PAGES 401-418

S 0033-569X(09)01111-5

Article electronically published on May 5, 2009

\title{
KINEMATICS OF A SHOCK WAVE OF ARBITRARY STRENGTH IN A NON-IDEAL GAS
}

\author{
BY \\ MANOJ PANDEY (Department of Mathematics, I.I.T. Bombay, Powai Mumbai, India 400076) \\ AND \\ V. D. SHARMA (Department of Mathematics, I.I.T. Bombay, Powai Mumbai, India 400076 )
}

\begin{abstract}
Singular surface theory is used to study the evolutionary behaviour of an unsteady three-dimensional motion of a shock wave of arbitrary strength propagating through a non-ideal gas. The dynamical coupling between the shock front and the induced discontinuities behind it is investigated by considering an infinite system of transport equations governing the strength of a shock wave and the induced discontinuities behind it. This infinite system, when subjected to a truncation approximation, efficiently describes the shock motion. Disturbances propagating on the shock and the onset of shock-shocks are briefly discussed. For a two-dimensional shock motion, our transport equations bear a structural resemblance to those of geometrical shock dynamics. Attention is drawn to the connection between the transport equation obtained by using the truncation rule and the one obtained by using the characteristic rule. The effects of van der Waals' excluded volume and wavefront geometry on the evolutionary behaviour of shocks are discussed.
\end{abstract}

1. Introduction. The problem of determining the motion of a shock wave of arbitrary strength has received considerable attention in the literature, and it has been a subject of great interest form both mathematical and physical points of view. If the shock is weak enough for the entropy changes across it to be negligible, a number of analytical approximations, such as the simple wave method of Friedrichs and the method of perturbation of characteristics of Lin (see [1] and 2]) offer a solution to the problem; but all these methods are low-order approximations and lose accuracy with increasing Mach number. Other methods by which the rise of entropy across the shock can be accounted for approximately have been proposed by Meyer [3], Ardavan-Rhad [4, Sirovich

Received March 21, 2007.

2000 Mathematics Subject Classification. Primary 35L50, 35L67, 35L65, 76L05.

Key words and phrases. Singular surface theory, non-ideal gas, shock wave, geometrical shock dynamics. Research support from ISRO-IIT Bombay, Space Technology Cell (Ref. No. 05-IS001) is gratefully acknowledged.

E-mail address: mkp@math.iitb.ac.in

E-mail address: vsharma@maths.iitb.ac.in 
and Chong [5] and Sharma et al. [6]. Indeed, the determination of the shock motion requires the calculation of the flow in the region behind the shock using methods generally belonging to the shock expansion theory. An interesting study on the exact numerical solutions of the gasdynamic equations has been carried out by Sirovich and his co-workers; for this, the reader is referred to the illuminating papers of Chong and Sirovich [7] and Lewis and Sirovich [8]. The method of intrinsic characterization of shock wave propagation avoids the cumbersome task of solving the gasdynamics equations governing the flow in the region behind the shock. An approximate theory of shock propagation, analogous to geometrical acoustics, has been developed by Whitham [9. In this theory, called geometrical shock dynamics, the motion of the shock can be determined without explicitly calculating the flow behind; the method, in fact, provides an efficient tool for approximately solving problems of shock propagation, involving diffraction (Collins and Chen [10, 11]), refraction (Catherasoo and Sturtevant [12]), focusing (Henshaw et al. [13], Schwendeman [14] and Cates and Sturtevant [15]) and stability (Schwendeman [16]).

The present work, which deals with the unsteady shock motion in three dimensions, derives motivation from the study relating to an intrinsic description of shock wave propagation carried out by Nunziato and Walsh [17, Chen [18] and Wright [19. A rigorous mathematical approach which can be used for describing kinematics of a shock of arbitrary strength has been proposed by Maslov [20, within the context of a weak shock, by using the theory of generalized functions to derive an infinite system of identities that hold on the shock front. Different methods for studying weak shocks have been proposed by Grinfeld [21, Anile and Russo 22] and Fu and Scott 23]. Using a procedure based on the kinematics of one-dimensional motion, Sharma and Radha 24] and Ravindran 25] studied the behaviour of one-dimensional shock waves in inhomogeneous atmospheres. An approximate analytical method for determining the evolutionary behavior of a bore of arbitrary strength as it approaches the shoreline on a sloping beach has been studied by Radha et al. [26, who derive identity relationships that hold on the bore front. The solution of the truncated finite system efficiently describes the shock motion. Recently, Batt and Ravindran [27] have provided a rigorous justification of this approximation within the context of the inviscid Burgers equation, which has certain features in common with the system appearing in this paper, where we use the singular surface theory to represent the shock as a propagating singular surface and study its evolutionary behaviour by considering a sequence of transport equations for the variation of jumps in pressure and its space derivatives across the shock. Our basic transport equations for a two-dimensional shock motion, which bear a close structural resemblance with those of geometrical shock dynamics [9, enable us to describe the onset of shock-shock and determine the effects of van der Waals' excluded volume and the wavefront geometry on the evolutionary behaviour of shock waves.

2. Basic equations and compatibility conditions. We consider a threedimensional shock wave propagating into a van der Waals gas region with varying density, velocity and pressure. The conservation laws for mass, momentum and energy may 
be expressed in the form

$$
\begin{aligned}
& \frac{\partial \rho}{\partial t}+\nabla \cdot(\rho \mathbf{v})=0 \\
& \frac{\partial \mathbf{v}}{\partial t}+(\mathbf{v} \cdot \nabla) \mathbf{v}+\rho^{-1} \nabla p=0 \\
& \frac{\partial p}{\partial t}+(\mathbf{v} \cdot \nabla p)+\frac{\gamma p}{(1-b \rho)}(\nabla \cdot \mathbf{v})=0
\end{aligned}
$$

where $\rho$ is the gas density, $\mathbf{v}=\left(v_{1}, v_{2}, v_{3}\right)$ is fluid velocity, $p$ is pressure and $\gamma$ is the specific heat ratio. We assume that the gas obeys a van der Waals equation of state of the form

$$
p(1-b \rho)=\rho R T,
$$

where $b$ the van der Waals excluded volume, $R$ is the gas constant and $T$ is the gas temperature. The shock is propagating through a given state $\mathbf{v} \equiv \mathbf{0}, p=p_{0}=$ constant, $\rho=\rho_{0}(\mathbf{x})$ where $\mathbf{x}=\left(x_{1}, x_{2}, x_{3}\right)$. Let the shock at any time $t$ and position $\mathbf{x}$ be given by

$$
F(t, \mathbf{x})=0 .
$$

Across a shock, the jump in a physical quantity is denoted by $[\psi]=\psi^{-}-\psi^{+}$, where $\psi^{+}$and $\psi^{-}$are values of $\psi$ immediately ahead of and just behind the shock surface respectively.

Let $U$ be the normal speed of the shock surface with $\mathbf{n}$ as its unit normal. Then it follows from (2.2) that

$$
U=-\frac{\partial F / \partial t}{\|\nabla F\|} ; \mathbf{n}=\frac{\nabla F}{\|\nabla F\|}
$$

Across the shock wave, the conservation laws of mass, momentum and energy yield the following jump relations

$$
[\mathbf{v}]=\frac{\left(a_{0}^{+}\right)^{2} \zeta\left(1-b \rho_{0}^{+}\right)}{\gamma U} \mathbf{n},[\rho]=\frac{2 \rho_{0}^{+}\left(1-b \rho_{0}^{+}\right) \zeta}{2 \gamma+(\gamma-1) \zeta+2 b \rho_{0}^{+} \zeta}, \zeta=\frac{[p]}{p_{0}^{+}},
$$

where

$$
U^{2}=\left(a_{0}^{+}\right)^{2}\left\{1+\frac{(\gamma+1) \zeta}{2 \gamma}\right\}
$$

with $\left(a_{0}^{+}\right)^{2}=\gamma p_{0}^{+} / \rho_{0}^{+}\left(1-b \rho_{0}^{+}\right)$as the equilibrium sound speed. In addition, we have the following compatibility conditions [28, which hold on the shock front:

$$
[\partial \psi / \partial t]=\partial[\psi] / \partial \tau-U[\mathbf{n} \cdot \nabla \psi],[\nabla \psi]=\mathbf{n}[(\mathbf{n} \cdot \nabla) \psi]+\tilde{\nabla}[\psi],
$$

where $\partial / \partial \tau$ and $\tilde{\nabla}$ denote respectively the temporal rate of change following the shock and the spatial rate of change along the shock surface defined as

$$
\partial \psi / \partial \tau=\partial \psi / \partial t+U(\mathbf{n} \cdot \nabla \psi), \tilde{\nabla} \psi=\nabla \psi-\mathbf{n}(\mathbf{n} \cdot \nabla) \psi .
$$

It immediately follows from $(2.7)_{2}$ that

$$
\mathbf{n} \cdot \tilde{\nabla} \psi=0, \tilde{\nabla} \cdot \mathbf{n}=\nabla \cdot \mathbf{n} .
$$


Equations (2.4) imply the following relations on the shock:

$$
\begin{aligned}
\frac{\partial[\rho]}{\partial \tau}= & \frac{2 \zeta\left\{(2 \gamma+(\gamma-1) \zeta)\left(1-2 b \rho_{0}^{+}\right)-2 b^{2}\left(\rho_{0}^{+}\right)^{2} \zeta\right\}}{\left(2 \gamma+(\gamma-1) \zeta+2 b \rho_{0}^{+} \zeta\right)^{2}} \frac{\partial \rho_{0}^{+}}{\partial \tau} \\
& +\frac{4 \gamma \rho_{0}^{+}\left(1-b \rho_{0}^{+}\right)}{\left(2 \gamma+(\gamma-1) \zeta+2 b \rho_{0}^{+} \zeta\right)^{2}} \frac{\partial \zeta}{\partial \tau}, \\
\frac{\partial[\mathbf{v}]}{\partial \tau}= & \frac{(4 \gamma+(\gamma+1) \zeta)\left(1-b \rho_{0}^{+}\right) U \mathbf{n}}{(2 \gamma+(\gamma+1) \zeta)^{2}} \frac{\partial \zeta}{\partial \tau}-\frac{U \zeta \mathbf{n}}{\rho_{0}^{+}(2 \gamma+(\gamma+1) \zeta)} \frac{\partial \rho_{0}^{+}}{\partial \tau} \\
& +\frac{\left(a_{0}^{+}\right) \zeta\left(1-b \rho_{0}^{+}\right)}{\gamma U} \frac{\partial \mathbf{n}}{\partial \tau}, \\
\frac{\partial[p]}{\partial \tau}= & p_{0}^{+} \frac{\partial \zeta}{\partial \tau} .
\end{aligned}
$$

3. Evolution equation for shock strength. Taking a jump in Equations (2.1) across the shock and using (2.6) and (2.8), we get

$$
\begin{aligned}
\frac{\partial[\rho]}{\partial \tau}+ & ([\mathbf{v}] \cdot \mathbf{n}-U)[\mathbf{n} \cdot \nabla \rho]+\left(\rho_{0}^{+}+[\rho]\right)\{\mathbf{n} \cdot[(\mathbf{n} \cdot \nabla) \mathbf{v}]+\tilde{\nabla} \cdot[\mathbf{v}]\} \\
+ & {[\mathbf{v}] \cdot\left(\nabla \rho_{0}^{+}\right)=0, } \\
\frac{\partial[\mathbf{v}]}{\partial \tau}+ & ([\mathbf{v}] \cdot \mathbf{n}-U)[(\mathbf{n} \cdot \nabla) \mathbf{v}]+\left(\rho_{0}^{+}+[\rho]\right)^{-1}\{\mathbf{n}[\mathbf{n} \cdot \nabla p]+\tilde{\nabla}[p]\}=0, \\
\frac{\partial[p]}{\partial \tau}+ & ([\mathbf{v}] \cdot \mathbf{n}-U)[\mathbf{n} \cdot \nabla p]+\gamma\left(\left[\frac{p}{1-b \rho}\right]+\frac{p_{0}^{+}}{1-b \rho_{0}^{+}}\right) \\
& \{\mathbf{n} \cdot[(\mathbf{n} \cdot \nabla) \mathbf{v}]+\tilde{\nabla} \cdot[\mathbf{v}]\}=0 .
\end{aligned}
$$

Now eliminating $[(\mathbf{n} \cdot \nabla) \mathbf{v}]$ between $(3.1)_{2}$ and $(3.1)_{3}$ and then using $(2.6)_{2}$ and $(2.9)_{2,3}$ we get the following nonlinear differential equation for the shock strength $\zeta$ :

$$
\frac{\partial \zeta}{\partial \tau}+w_{1} \nabla \cdot \mathbf{n}=-w_{2}(\mathbf{n} \cdot \nabla p)^{-}+w_{3}\left(\mathbf{n} \cdot \nabla \rho_{0}^{+}\right),
$$

where

$$
\begin{aligned}
& w_{1}=\left\{2 \gamma U \zeta(1+\zeta)(2 \gamma+(\gamma-1) \zeta)+2 b \rho_{0}^{+}\right\} / \Pi, \\
& w_{2}=\left\{(\gamma+1) U \zeta\left(2 \gamma+(\gamma-1) \zeta+2 b \rho_{0}^{+} \zeta\right)\right\} / \Pi p_{0}^{+}, \\
& w_{3}=\{\gamma U \zeta(1+\zeta)(2 \gamma+(\gamma+1) \zeta)\} /\left\{\rho_{0}^{+}\left(1-b \rho_{0}^{+}\right) \Pi\right\}, \\
& \Pi=8 \gamma^{2}+\left(9 \gamma^{2}+\gamma\right) \zeta+(2 \gamma-1)(\gamma+1) \zeta^{2} .
\end{aligned}
$$

The evolutionary behaviour of a shock wave propagating into an inhomogeneous medium is governed by (3.2). In equation (3.2), the term $\nabla \cdot \mathbf{n}$ equals twice the mean curvature of the shock surface, and the term $(\mathbf{n} \cdot \nabla p)^{-}$, which is still unknown, represents the effect of induced discontinuities or disturbances that overtake the shock from behind. Equations (2.3) imply that

$$
\begin{aligned}
& \frac{\partial \mathbf{n}}{\partial t}=\frac{\partial(\nabla F) / \partial t}{\|\nabla F\|}-\frac{\mathbf{n}(\mathbf{n} \cdot \nabla)(\partial F / \partial t)}{\|\nabla F\|}, \\
& \frac{\partial \mathbf{n}}{\partial x_{j}}=\frac{\partial(\nabla F) / \partial x_{j}}{\|\nabla F\|}-\frac{\mathbf{n}(\mathbf{n} \cdot \nabla)\left(\partial F / \partial x_{j}\right)}{\|\nabla F\|},
\end{aligned}
$$


which on using (2.5) and definitions of $\partial / \partial \tau$ and $\tilde{\nabla}$, yield

$$
\frac{\partial \mathbf{n}}{\partial \tau}+\frac{(\gamma+1)\left(a_{0}^{+}\right)^{2}}{4 \gamma U} \nabla \zeta=\frac{\left(1-2 b \rho_{0}^{+}\right) U}{2 \rho_{0}^{+}\left(1-b \rho_{0}^{+}\right)} \nabla \rho_{0}^{+} .
$$

From the definition of $\partial / \partial \tau$, it follows that the instantaneous motion of the shock surface is described by the relation

$$
\frac{\partial \mathbf{x}}{\partial \tau}=U \mathbf{n} .
$$

Equations (3.3) and (3.4) describe the shock rays, which are along the normal and form a family of orthogonal trajectories to the successive positions of the shock surface. Thus, the successive positions of the shock front, its orientation and the distribution of shock strength $\zeta$ on it, can be computed from (3.2), (3.3) and (3.4) provided the term $(\mathbf{n} \cdot \nabla p)^{-}$, which provides a coupling of the shock with the flow behind the shock, is made known. Thus, the equations (3.2), (3.3) and (3.4) show that the evolutionary behaviour of the shock strength at any time $\tau$ depends not only on the strength of the shock, its normal and mean curvature but it also depends on the inhomogeneities present in the medium ahead of the shock, the van der Waals excluded volume $b$ and the normal pressure gradient immediately behind the wave at time $\tau$; the situation $b=0$ corresponds to an ideal gas.

4. Wave propagation on the shock. The unit normal $\mathbf{n}$ to the shock surface, and its divergence can be expressed as

$$
\mathbf{n}=\{\cos \theta \sin \phi, \sin \theta \sin \phi, \cos \phi\}, \nabla \cdot \mathbf{n}=\sin \phi\left(\partial \theta / \partial \eta_{2}\right)-\left(\partial \phi / \partial \eta_{1}\right),
$$

where $\theta$ and $\phi$ are the angles which the normal $\mathbf{n}$ makes with the $x_{1}$ and $x_{3}$ axes respectively, with

$$
\begin{aligned}
\partial / \partial \eta_{1} & =\{-\cos \theta \cos \phi,-\sin \theta \cos \phi, \sin \phi\} \cdot \nabla, \\
\partial / \partial \eta_{2} & =\{-\sin \theta, \cos \theta, 0\} \cdot \nabla,
\end{aligned}
$$

being the spatial rates of change along two mutually orthogonal directions tangential to the shock surface. In view of (4.1) and (4.2), the shock strength equation (3.2) and the shock ray equations (3.3) and (3.4) become

$$
\begin{aligned}
& \frac{\partial \zeta}{\partial \tau}+w_{1}\left(\sin \phi \frac{\partial \theta}{\partial \eta_{2}}-\frac{\partial \phi}{\partial \eta_{1}}\right)=-w_{2}(\mathbf{n} \cdot \nabla p)^{-}+w_{3}\left(\mathbf{n} \nabla \rho_{0}^{+}\right), \\
& \frac{\partial \theta}{\partial \tau}+\frac{(\gamma+1)\left(a_{0}^{+}\right)^{2}}{4 \gamma U \sin \phi} \frac{\partial \zeta}{\partial \eta_{2}}=\frac{U\left(1-2 b \rho_{0}^{+}\right)}{2 \rho_{0}^{+}\left(1-b \rho_{0}^{+}\right) \sin \phi} \frac{\partial \rho_{0}^{+}}{\partial \eta_{2}} \\
& \frac{\partial \phi}{\partial \tau}-\frac{(\gamma+1)\left(a_{0}^{+}\right)^{2}}{4 \gamma U} \frac{\partial \zeta}{\partial \eta_{1}}=-\frac{U\left(1-2 b \rho_{0}^{+}\right)}{2 \rho_{0}^{+}\left(1-b \rho_{0}^{+}\right)} \frac{\partial \rho_{0}^{+}}{\partial \eta_{1}} \\
& \frac{\partial x_{1}}{\partial \tau}=U \cos \theta \sin \phi \\
& \frac{\partial x_{2}}{\partial \tau}=U \sin \theta \sin \phi \\
& \frac{\partial x_{3}}{\partial \tau}=U \cos \phi .
\end{aligned}
$$


Equations (4.3) together with the initial values of $\theta, \phi, \zeta$, and $\mathbf{x}$, specified as functions of $\eta_{1}$ and $\eta_{2}$ at $\tau=0$, allow us to determine the successive positions $\mathbf{x}$ of the shock front, the inclinations $\theta$ and $\phi$ of its normal, and the distribution of the strength $\zeta$ on the shock surface. The wave motion on the shock surface itself is brought out by studying the characteristic surfaces of equations (4.3), which can be written in the form

$$
\frac{\partial Y}{\partial \tau}+A^{\nu} \frac{\partial Y}{\partial \eta_{\nu}}=B \quad \nu=1,2
$$

where $Y=\left(\zeta, \theta, \phi, x_{1}, x_{2}, x_{3}\right)^{t r}$ is a column vector of unknowns, $A^{\nu}$ are $6 \times 6$ matrices, and $B$ is a column vector, which can be read off by inspection of (4.3). Here and throughout, summation over a repeated subscript is automatic unless otherwise indicated. Let $f\left(\eta_{1}, \eta_{2}, \tau\right)=0$ be the equation for the characteristic surface of (4.4) separating two regions in each of which $Y$ is continuously differentiable. If $\mathbf{N}=\left(N_{1}, N_{2}\right)$ denotes the unit normal to the characteristic surface in its direction of propagation, and if $S(>0)$ denotes its speed, then

$$
\mathbf{N}=\left(\nabla_{\eta} f\right) /\left\|\nabla_{\eta} f\right\|, S=-\left(\frac{\partial f}{\partial \tau}\right) /\left\|\nabla_{\eta} f\right\|,
$$

where $\nabla_{\eta}=\left\{\partial / \partial \eta_{1}, \partial / \partial \eta_{2}\right\}$; it can be easily shown that all possible speeds of propagation satisfy the characteristic condition

$$
\operatorname{det}\left(A^{\nu} N_{\nu}-S I\right)=0,
$$

where $I$ is a $6 \times 6$ unit matrix.

Equation (4.6) has six roots; the root $S=0$, which is of multiplicity four, corresponds to the requirement that the derivatives of $\zeta, \theta, \phi$, etc. are continuous on the shock surface. The other two non-zero characteristic roots, $S= \pm c^{1 / 2}$, where

$$
c=\frac{(\gamma+1)\left(a_{0}^{+}\right)^{2} \zeta(1+\zeta)\left(2 \gamma+(\gamma-1) \zeta+2 b \rho_{0}^{+}\right)}{\Pi},
$$

correspond to wave propagation on the shock surface. It is evident from (4.7) that the speed $S$ of the wave propagation is independent of the direction $\mathbf{N}$.

5. Shock-shocks. Let us consider the characteristic surface $f\left(\eta_{1}, \eta_{2}, \tau\right)=0$, which is propagating with the speed $S=c^{1 / 2}$. Our convention is that $S>0$ corresponds to the surface $f=0$ propagating in the direction of $\mathbf{N}$. Then across $f=0$, the variables $\zeta, \theta, \phi$, etc. are continuous while their first and second order partial derivatives with respect to $\eta_{\nu}$ and $\tau$ suffer jump discontinuities. If the jump across $f=0$ in an entity $\psi\left(\eta_{1}, \eta_{2}, \tau\right)$ is denoted by $[\psi]$, where the brackets stand for the quantity enclosed immediately behind the wave front $f=0$ minus its value just ahead of $f=0$, which shall be denoted by $\psi_{*}$, then the first and second-order compatibility conditions, which hold across $f=0$ are 28.

$$
\begin{aligned}
& {[\partial \psi / \partial \tau]=-S \hat{\psi},\left[\nabla_{\eta} \psi \rrbracket=\mathbf{N} \hat{\psi},\right.} \\
& {\left[\nabla_{\eta}(\partial \psi / \partial \tau) \rrbracket=-S \bar{\psi} \mathbf{N}-S\left(\tilde{\nabla}_{\eta} \hat{\psi}\right)+(d \hat{\psi} / d \tau) \mathbf{N}+\hat{\psi}(d \mathbf{N} / d \tau),\right.} \\
& {\left[\partial^{2} \psi / \partial \eta_{\nu} \partial \eta_{\delta} \rrbracket=N_{\nu} N_{\delta} \bar{\psi}+N_{\delta} \tilde{\partial} \hat{\psi} / \partial \eta_{\nu}+N_{\nu} \tilde{\partial} \hat{\psi} / \partial \eta_{\delta}+\hat{\psi} \tilde{\partial} N_{\nu} / \partial \eta_{\delta},\right.}
\end{aligned}
$$


where

$$
\begin{aligned}
& \hat{\psi}=\left[\mathbf{N} \cdot \nabla_{\eta} \psi\right], \bar{\psi}=\left[N_{\nu} N_{\delta} \partial^{2} \psi / \partial \eta_{\nu} \partial \eta_{\delta}\right], d / d \tau=\partial / \partial \tau+S\left(\mathbf{N} \cdot \nabla_{\eta}\right) \\
& \tilde{\nabla}_{\eta}=\nabla_{\eta}-\mathbf{N}\left(\mathbf{N} \cdot \nabla_{\eta}\right), d \mathbf{N} / d \tau=-\left(\tilde{\nabla}_{\eta} S\right)_{Y=Y_{*}}, \tilde{\partial} / \partial \eta_{\nu}=\partial / \partial \eta_{\nu}-N_{\nu}\left(\mathbf{N} \cdot \nabla_{\eta}\right)
\end{aligned}
$$

Forming jumps across $f=0$ in equations (4.3), we obtain on using (5.1) that

$$
\hat{\theta}=\frac{\Gamma_{*}}{S \sin \phi_{*}} N_{2} \hat{\zeta}, \hat{\phi}=-\frac{\Gamma_{*}}{S} N_{1} \hat{\zeta}, \hat{\mathbf{x}}=0
$$

where $\Gamma_{*}=(\gamma+1)\left(a_{0}^{+}\right)_{*}^{2} / 4 \gamma U$. Following a standard procedure, if we differentiate equations (4.3) with respect to $\eta_{\nu}$, form jumps across $f=0$ in the resulting equations, make use of the compatibility conditions (5.1) and the relations (5.2), and then eliminate quantities with overhead bars, we obtain the following equation in $\hat{\zeta}$ on $f=0$ :

$$
\frac{d \hat{\zeta}}{d \tau}+Q \hat{\zeta}+R \hat{\zeta}^{2}=0
$$

where $R=\left(S_{*} / 2\right)\left(\partial\left(\ln w_{1}\right) / \partial \zeta\right)_{*}-\left\{c_{*}^{3 / 2} /\left(2 w_{1_{*}} U_{*}\right)\right\}$ and $Q=P+d\left(\ln \Theta^{1 / 2}\right) / d \tau$ with $\Theta=c_{*}^{1 / 2} /\left\{w_{1_{*}}\left(\sin \phi_{*}\right)^{N_{2}^{2}}\right\}$,

$$
\begin{aligned}
& P=(1 / 2) S_{*}\left(\partial N_{\nu} / \partial \eta_{\nu}\right)+S_{*} / 2\left\{\cot \phi_{*} N_{2}^{2}\left(\mathbf{N} \cdot \nabla_{\eta} \phi\right)_{*}-\cot \phi_{*} N_{2}\left(\partial \phi / \partial \eta_{2}\right)_{*}\right. \\
& -N_{1} \cos \phi_{*}\left(\partial \theta / \partial \eta_{2}\right)_{*}+\left(\mathbf{N} \cdot \nabla_{\eta} \ln w_{1}\right)_{*}+\left(\partial\left(\ln w_{1}\right) / \partial \zeta\right)_{*}\left(\mathbf{N} \cdot \nabla_{\eta} \zeta\right)_{*} \\
& -\left(N_{2} / w_{1_{*}} \sin \phi_{*}\right)\left(\partial G_{1} / \partial \theta\right)_{*}+\left(N_{1} / w_{1_{*}}\right)\left(\partial G_{1} / \partial \phi\right)_{*}+\Gamma_{*} \cot \phi_{*} N_{1} N_{2}\left(\partial \zeta / \partial \eta_{2}\right)_{*} \\
& +2(\partial(\ln \Gamma) / \partial \zeta)_{*}\left(\mathbf{N} \cdot \nabla_{\eta} \zeta\right)_{*}-\sin \phi_{*} N_{2}\left(\partial G_{2} / \partial \zeta\right)_{*}+\left(\mathbf{N} \cdot \nabla_{\eta} \ln \Gamma\right)_{*} \\
& \left.+\left(N / \Gamma_{*}\right)\left(\partial G_{3} / \partial \zeta\right)_{*}\right\}+(1 / 2)\left\{\sin \phi_{*}\left(\partial w_{1} / \partial \zeta\right)_{*}\left(\partial \theta / \partial \eta_{2}\right)_{*}-\left(\partial w_{1} / \partial \zeta\right)_{*}\left(\partial \phi / \partial \eta_{1}\right)_{*}\right. \\
& -N_{2}^{2}\left(\partial G_{2} / \partial \theta\right)_{*}+N_{1} N_{2} \sin \phi_{*}\left(\partial G_{2} / \partial \phi\right)_{*}+\left(N_{1} N_{2} \sin \phi_{*}\right)\left(\partial G_{3} / \partial \theta\right)_{*} \\
& \left.-N_{1}^{2}\left(\partial G_{3} / \partial \phi\right)_{*}-\left(\partial G_{1} / \partial \zeta\right)_{*}\right\}
\end{aligned}
$$

and

$$
\begin{aligned}
G_{1} & =-w_{2}(\mathbf{n} \cdot p)+w_{3}\left(\mathbf{n} \cdot \nabla \rho_{0}^{+}\right), G_{2}=\frac{U\left(1-2 b \rho_{0}^{+}\right)}{2 \rho_{0}^{+}\left(1-b \rho_{0}^{+}\right) \sin \phi} \frac{\partial \rho_{0}^{+}}{\partial \eta_{2}} \\
G_{3} & =-\frac{U\left(1-2 b \rho_{0}^{+}\right)}{2 \rho_{0}^{+}\left(1-b \rho_{0}^{+}\right)} \frac{\partial \rho_{0}^{+}}{\partial \eta_{1}}
\end{aligned}
$$

A thorough treatment of the local and global properties of the solution of an equation such as (5.3) within a general framework has been given by Menon et al. 29] and Sharma and Menon [30]. Equation (5.3) can be integrated to yield

$$
\hat{\zeta}=\frac{\hat{\zeta}(0)\left(\Theta / \Theta_{0}\right)^{-1 / 2} \exp \left(-\int_{0}^{\tau} P(t) d t\right)}{1+\Theta_{0}^{1 / 2} \hat{\zeta}(0) J(\tau)},
$$


where $J(\tau)=\int_{0}^{\tau}\left(R / \Theta^{1 / 2}\right) \exp \left(-\int_{0}^{t} P\left(\tau^{\prime}\right) d \tau^{\prime}\right) d t$, and $\hat{\zeta}(0)$ indicates the value of $\hat{\zeta}$ at $\tau=0$. Thus, when both $Q(\tau)$ and $R(\tau)$ are integrable on all finite sub-intervals of $[0, \infty)$ and the function $R(\tau)$ is of constant sign on $[0, \infty)$, there are two possible situations described below:

(i) If $\operatorname{sgn} \hat{\zeta}(0)=\operatorname{sgn} R(\tau)$, then the denominator of (5.4) must either approach a finite limit or $\infty$ as $\tau \rightarrow \infty$, and thus a shock discontinuity on the original shock, referred to as "shock-shock", cannot form. Clearly, $\hat{\zeta}$ is finite, nonzero, and continuous on $[0, \infty)$, and therefore the behaviour of $\hat{\zeta}$ as $\tau \rightarrow \infty$ is determined by the sign of $Q$ in (5.3); thus for sufficiently large $\tau, Q>0$ and $\liminf _{\tau \rightarrow \infty} \Theta \neq 0$, $\hat{\zeta} \rightarrow 0$ as $\tau \rightarrow \infty$, i.e. the wave on the original shock decays and dies out eventually. Similarly, if for sufficiently large $\tau, Q<0, \liminf _{\tau \rightarrow \infty} \Theta \neq 0$ and $(-Q / R)$ tends to a finite or infinite limit $l$ as $\tau \rightarrow \infty$, then $\lim _{\tau \rightarrow \infty} \hat{\zeta}=l$; while if $(-Q / R)$ is bounded below, then so is $\hat{\zeta}$.

(ii) However, if $\operatorname{sgn} \hat{\zeta}(0)=-\operatorname{sgn} R(\tau)$, then there are three possibilities:

(a) When $|\hat{\zeta}(0)|<\hat{\zeta}_{c}$, where $\hat{\zeta}_{c}=\left(\Theta_{0}^{1 / 2} J(\infty)\right)^{-1}$, then $\hat{\zeta}$ is finite, nonzero, and continuous over $[0, \infty)$ and $|\hat{\zeta}| \rightarrow 0$ or $\infty$ if and only if $\Theta^{-1 / 2} \exp \left(-\int_{0}^{\tau} P(t) d t\right) \rightarrow 0$, or, $\infty$ respectively as $\tau \rightarrow \infty$.

(b) When $|\hat{\zeta}(0)|<\hat{\zeta}_{c}$, then there exists a finite time $\tau_{c}>0$, given by $J\left(\tau_{c}\right)=$ $1 /\left(\Theta_{0}^{1 / 2}|\hat{\zeta}(0)|\right)$, such that $\hat{\zeta}$ is finite, nonzero, and continuous on $\left[0, \tau_{c}\right)$ and $|\hat{\zeta}| \rightarrow \infty$ as $\tau \rightarrow \tau_{c}$. This signifies the appearance of a "shock-shock" at an instant $\tau_{c}$.

(c) However, when $|\hat{\zeta}(0)|=\left(\Theta_{0}^{1 / 2} J(\infty)\right)^{-1}$, then $\hat{\zeta}$ is finite, nonzero, and continuous over $[0, \infty)$, and if $\liminf _{\tau \rightarrow \infty} \Theta \neq 0$ and $(-Q / R)$ tends to a finite nonzero or infinite limit $l$ as $\tau \rightarrow \infty$, then $\lim _{\tau \rightarrow \infty}=l$. Notice that it is possible for $\hat{\zeta}_{c}$ to vanish; for instance, $\hat{\zeta}_{c}=0$ if $\liminf _{\tau \rightarrow \infty} \Theta \neq 0$ and $\liminf _{\tau \rightarrow \infty}\left\{\Theta^{-1 / 2} \exp \left(-\int_{0}^{\tau} P(t) d t\right)\right\} \neq 0$. In this case a "shock-shock" is always formed if $\hat{\zeta}(0)<0$.

6. Two-dimensional configuration. Setting $\phi=\pi / 2$ and $\partial / \partial x_{3}=0$ in equations (4.3), the shock wave propagation in the $x_{1}, x_{2}$ plane can be described by the following equations:

$$
\begin{aligned}
& \frac{\partial \theta}{\partial \eta}+\frac{U}{w_{1}} \frac{\partial \zeta}{\partial \sigma}=\frac{(2 \gamma+(\gamma-1) \zeta)}{2 \rho_{0}^{+}\left(1-b \rho_{0}^{+}\right)\left(2 \gamma+(\gamma-1) \zeta+2 b \rho_{0}^{+} \zeta\right)}\left(\frac{\partial \rho_{0}^{+}}{\partial n}\right) \\
&-\frac{(\gamma+1)}{2 \gamma(1+\zeta) p_{0}^{+}}\left(\frac{\partial p}{\partial n}\right)^{-}, \\
& \frac{\partial \theta}{\partial \sigma}+ \frac{(\gamma+1)\left(a_{0}^{+}\right)^{2}}{4 \gamma U^{2}} \frac{\partial \zeta}{\partial \eta}=\frac{1-2 b \rho_{0}^{+}}{2 \rho_{0}^{+}\left(1-b \rho_{0}^{+}\right)} \frac{\partial \rho_{0}^{+}}{\partial \eta}, \\
& \frac{\partial x_{1}}{\partial \sigma}=\cos \theta, \\
& \frac{\partial x_{2}}{\partial \sigma}=\sin \theta,
\end{aligned}
$$


where $d \sigma=U d \tau$ is the distance traveled by the shock in time $d \tau$ along the shock ray, $d \eta \equiv d \eta_{2}$ is the distance measured along the shock front, and the derivative $(\partial p / \partial n)^{-}$ refers to the instantaneous value of the space derivative of $p$ behind the shock along its normal. Thus, the successive shock positions and the rays, described by the family of curves $\sigma=$ constant and $\eta=$ constant, respectively, form an orthogonal coordinate system $(\sigma, \eta)$, which is exactly the same as the orthogonal system $(\alpha, \beta)$ introduced in the theory of geometrical shock dynamics 9 . Thus, the line element along the two families of curves are

$$
d \sigma=M d \alpha \text { and } d \eta=A d \beta
$$

where $M=U / a_{0}$ is the shock Mach number, and $A(\alpha, \beta)$ is the ray-tube along the shock ray that satisfies the relation

$$
\frac{\partial A}{\partial \tau}=A U(\nabla \cdot \mathbf{n})
$$

which for the two-dimensional problem under consideration, becomes

$$
\frac{\partial A}{\partial \sigma}=A\left(\frac{\partial \theta}{\partial \eta}\right)
$$

and assumes the following form when expressed in the $(\alpha, \beta)$ coordinates [9]:

$$
\frac{\partial \theta}{\partial \beta}=M^{-1} \frac{\partial A}{\partial \alpha}
$$

In view of (6.2), the shock strength equation $(6.1)_{1}$ in the $(\alpha, \beta)$ coordinates becomes

$$
\frac{\partial \theta}{\partial \beta}+\frac{A g(M)}{M^{2}-1} \frac{\partial M}{\partial \alpha}=A \omega\left(\frac{\partial p}{\partial n}\right)^{-}+A \chi\left(\frac{\partial \rho_{0}^{+}}{\partial n}\right)
$$

where

$$
\begin{aligned}
& g(M)=\frac{(\gamma+1)\left\{4(\gamma+1)+\left(9 \gamma^{2}+\gamma\right)\left(M^{2}-1\right)+2(2 \gamma-1)\left(M^{2}-1\right)^{2}\right\}}{\left(1+\left(2 M^{2}-1\right) \gamma\right)\left(2+(\gamma-1) M^{2}+2 b \rho_{0}^{+}\left(M^{2}-1\right)\right)}, \\
& \omega=-\frac{(\gamma+1)^{2}}{2 \rho_{0}^{+}\left(1-b \rho_{0}^{+}\right)\left(a_{0}^{+}\right)^{2}\left(1+\left(2 M^{2}-1\right) \gamma\right)}, \\
& \chi=\frac{2+(\gamma-1) M^{2}}{2 \rho_{0}^{+}\left(1-b \rho_{0}^{+}\right)\left(2+(\gamma-1) M^{2}+2 b \rho_{0}^{+}\left(M^{2}-1\right)\right)} .
\end{aligned}
$$


If we use (6.3) and (6.4), we obtain the following differential equation connecting the variation of $A$ and $M$ along the rays:

$$
\frac{1}{A} \frac{\partial A}{\partial \alpha}+\frac{M g(M)}{\left(M^{2}-1\right)} \frac{\partial M}{\partial \alpha}=M \omega\left(\frac{\partial p}{\partial n}\right)^{-}+M \chi\left(\frac{\partial \rho_{0}^{+}}{\partial n}\right) .
$$

Similarly equations (6.1) ${ }_{2}-(6.1)_{3}$, in terms of the $(\alpha, \beta)$ coordinates and the Mach number $M$, become

$$
\begin{aligned}
& \frac{\partial \theta}{\partial \alpha}+\frac{1}{A} \frac{\partial M}{\partial \beta}=0 \\
& \frac{\partial x_{1}}{\partial \alpha}=M \cos \theta \\
& \frac{\partial x_{2}}{\partial \alpha}=M \sin \theta
\end{aligned}
$$

It may be noticed that in the absence of the coupling term $(\partial p / \partial n)^{-}$, equations $(6.3)-$ (6.6) form a complete set to determine $A, M, \theta$, and $\mathbf{x}$; indeed, when $b=0$ (i.e. an ideal gas case) and $\rho_{0}^{+}=$constant, the transport equations (6.3) and $(6.6)_{1,2,3}$ bear a close structural resemblance to those of geometrical shock dynamics. Here $M$ lies in the interval $1<M<\infty$; small values of $M-1$ correspond to weak shocks and large values to strong shocks. It may be noticed further that for a weak shock $g(M) \rightarrow 4$ as $M \rightarrow 1$, and, consequently, the corresponding weak wave approximation of (6.5) that is obtained by neglecting the coupling term coincides exactly with that of geometrical shock dynamics 9. It is interesting to note that an area-Mach number relation similar to (6.5) for the case of shock propagation in an ideal gas with nonuniform propagation ahead was considered previously by Catherasoo and Sturtevant [12] and Schwendeman 14.

7. Transport equations for the coupling term $(\mathbf{n} \cdot \nabla p)^{-}$. Since the coupling term $(\mathbf{n} \cdot \nabla p)^{-}$is an unknown, we need to obtain a transport equation for it; to achieve this goal, we proceed as follows. In order to make algebraic calculation less cumbersome, we consider the state ahead of the shock, $F(t, \mathbf{x})=0$, to be uniform and at rest, and $\|\nabla F\|=1$. The transport equation (3.2) for the shock strength then becomes

$$
\frac{\partial \zeta}{\partial \tau}+w_{1} \nabla \cdot \mathbf{n}=-w_{2}(\mathbf{n} \cdot \nabla p)^{-} .
$$

In view of the fact that $\mathbf{v}_{0}^{+}=0$ and $\|\nabla F\|=1$, it is readily shown from (2.3) that

$$
\mathbf{n} \cdot \nabla \zeta=0
$$

Differentiating (7.2) with respect to $\tau$ and using (7.1) in the resulting equation, we get

$$
\frac{(\gamma+1) U^{3}}{4\left(a_{0}^{+}\right)^{2}(1+\zeta)^{2}\left(1-b \rho_{0}^{+}\right)}(\nabla \zeta)^{2}=w_{1} \mathbf{n} \cdot \nabla(\nabla \cdot \mathbf{n})-w_{2} \mathbf{n} \cdot \nabla(\mathbf{n} \cdot \nabla p)^{-} .
$$


In view of (2.9) $2,(3.3),(7.1)$, and (7.2), equation (3.1) 2 yields

$$
((\mathbf{n} \cdot \nabla) \mathbf{v})^{-}=\epsilon_{1} \mathbf{n}(\mathbf{n} \cdot \nabla p)^{-}+\epsilon_{2} \mathbf{n}(\nabla \cdot \mathbf{n})+\epsilon_{3} \nabla \zeta
$$

where

$$
\begin{aligned}
& \epsilon_{1}=\left\{16 \gamma^{2}+2\left(7 \gamma^{2}-\gamma\right) \zeta+3\left(\gamma^{2}-1\right) \zeta^{2}\right\} / 2 U \rho_{0}^{+} \Pi, \\
& \epsilon_{2}=-\left\{2 \gamma U \zeta(1+\zeta)\left(1-b \rho_{0}^{+}\right)(4 \gamma+(\gamma+1) \zeta)\right\} /\{(2 \gamma(\gamma+1) \zeta) \Pi\}, \\
& \epsilon_{3}=\left\{\left(a_{0}^{+}\right)^{2}\left(1-b \rho_{0}^{+}\right)\left(4 \gamma+(\gamma-3) \zeta+2 b \rho_{0}^{+} \zeta\right)\right\} /\left\{2 \gamma U\left(2 \gamma+(\gamma-1) \zeta+2 b \rho_{0}^{+} \zeta\right)\right\},
\end{aligned}
$$

with $\Pi$ being the same as in (3.2). Similarly, equations $(3.1)_{1,2}$, in view of $(2.9)_{1,2,3}$, (17.1), and (7.4), yield

$$
(\mathbf{n} \cdot \nabla \rho)^{-}=\epsilon_{4}(\mathbf{n} \cdot \nabla p)^{-}+\epsilon_{5}(\nabla \cdot \mathbf{n}),
$$

where

$$
\begin{aligned}
\epsilon_{4} & =\frac{(2 \gamma+(\gamma+1) \zeta)^{2}\left(16 \gamma^{2}+2\left(5 \gamma^{2}-2 \gamma\right) \zeta+3\left(\gamma^{2}-1\right) \zeta^{2}\right)}{2 U \Pi\left(2 \gamma+(\gamma-1) \zeta+2 b \rho_{0}^{+} \zeta\right)^{2}} \\
\epsilon_{5} & =-\frac{2 \zeta\left(1-b \rho_{0}^{+}\right) \rho_{0}^{+}(2 \gamma+(\gamma+1) \zeta)\left(16 \gamma^{2}+2\left(9 \gamma^{2}+\gamma\right) \zeta+(3 \gamma-1)(\gamma+1) \zeta^{2}\right)}{\Pi\left(2 \gamma+(\gamma-1)+2 b \rho_{0}^{+}\right)^{2}}
\end{aligned}
$$

The second-order compatibility relations, which will be used in the sequel, are as follows 28]:

$$
\begin{aligned}
& {\left[\nabla\left(\frac{\partial \psi}{\partial t}\right)\right]=\mathbf{n} \frac{\partial[\mathbf{n} \cdot \nabla \psi]}{\partial t}+[\mathbf{n} \cdot \nabla \psi] \frac{\partial \mathbf{n}}{\partial t}-U \mathbf{n}\left[n_{i} n_{j} \frac{\partial^{2} \psi}{\partial x_{j} \partial x_{i}}\right]} \\
& +U\left(\tilde{\nabla}_{j} \mathbf{n}\right)\left(\tilde{\nabla}_{j}[\psi]\right)+\frac{\partial}{\partial \tau}\left(\tilde{\nabla}_{i}[\psi]\right), \\
& {\left[\frac{\partial^{2} \psi}{\partial x_{i} \partial x_{j}}\right]=\left(\tilde{\nabla}_{i} \tilde{\nabla}_{j}[\psi]\right)+\left(\tilde{\nabla}_{i} n_{j}\right)[\mathbf{n} \cdot \nabla \psi]+n_{j}\left(\tilde{\nabla}_{i}[\mathbf{n} \cdot \nabla \psi]\right)} \\
& +n_{i}\left(\tilde{\nabla}_{j}[\mathbf{n} \cdot \nabla \psi]\right)-n_{i}\left(\tilde{\nabla}_{j} n_{k}\right)\left(\tilde{\nabla}_{k}[\psi]\right)+n_{i} n_{j}\left[n_{l} n_{k} \frac{\partial^{2} \psi}{\partial x_{l} \partial x_{k}}\right],
\end{aligned}
$$

where $\tilde{\nabla}_{j}=\nabla_{j}-n_{j}(\mathbf{n} \cdot \nabla)$. Now we differentiate equations (2.1) with respect to $x_{k}$, take jumps in the resulting equations across the shock, multiply the equations by $n_{k}$, and make use of the compatibility relation (2.6), (7.6) and the equations (2.4), (2.9), (3.1), (7.1), (7.2), (7.3), (77.4), and (7.5) to get the following transport equation for the coupling term $\Lambda_{(1)}=(\mathbf{n} \cdot \nabla p)^{-}$:

$$
\frac{\partial \Lambda_{(1)}}{\partial \tau}+\varphi_{1} \Lambda_{(2)}+\varphi_{2} \Lambda_{(1)}^{2}+\varphi_{3} \Lambda_{(1)}+\varphi_{4}=0
$$


where $\Lambda_{(2)}$, defined as $\Lambda_{(2)}=\left(n_{i} n_{j} p_{, i j}\right)^{-}$, is the second-order coupling term and the coefficients $\varphi_{1}, \varphi_{2}, \varphi_{3}$, and $\varphi_{4}$ are given by

$$
\begin{aligned}
& \varphi_{1}=\left\{(\gamma+1) \zeta U\left(2 \gamma+(\gamma-1) \zeta+2 b \rho_{0}^{+} \zeta\right)\right\} /\{\lambda(2 \gamma+(\gamma+1) \zeta)(2 \gamma+(\gamma-1) \zeta)\}, \\
& \lambda=\left\{(2 \gamma+(\gamma+1) \zeta)+2 \gamma U \rho_{0}^{+}(1+\zeta) \epsilon_{1}\right\} /(2 \gamma+(\gamma+1) \zeta), \\
& \varphi_{2}=\frac{\epsilon_{1}}{\lambda}\left\{(\gamma+1)(2 \gamma+(\gamma-1) \zeta)-b \rho_{0}^{+}(2 \gamma+(\gamma+1) \zeta)\right\}\left\{\left(1-b \rho_{0}^{+}\right)(2 \gamma\right. \\
& +(\gamma-1) \zeta)\}^{-1}-\frac{\left(a_{0}^{+}\right)^{2} \rho_{0}^{+}(1+\zeta)}{\lambda U}\left\{w_{2} d \epsilon_{2} / d \zeta-\epsilon_{1}^{2}+\{2 \gamma+(\gamma-1) \zeta\right. \\
& \left.\left.+2 b \rho_{0}^{+} \zeta\right\}^{2}\left(\rho_{0}^{+}\right)^{-2}\{2 \gamma+(\gamma+1) \zeta\}^{-2} \epsilon_{4}\right\}+\frac{b \epsilon_{4}}{\lambda}\left\{\left(a_{0}^{+}\right)^{2} \rho_{0}^{+}(1+\zeta)(2 \gamma\right. \\
& \left.\left.+(\gamma-1) \zeta+2 b \rho_{0}^{+} \zeta\right)^{2}\right\}\left(1-b \rho_{0}^{+}\right)^{-1}\{2 \gamma+(\gamma-1) \zeta\}^{-2}, \\
& \varphi_{3}=\frac{\nabla \cdot \mathbf{n}}{\lambda}\left\{\epsilon_{2}+\left(a_{0}^{+}\right)^{2}(1+\zeta) \epsilon_{1}\left\{2 \gamma+(\gamma-1) \zeta+2 b \rho_{0}^{+} \zeta\right\}\{2 \gamma\right. \\
& +(\gamma-1) \zeta\}^{-1}\left(1-b \rho_{0}^{+}\right)^{-1}+b \epsilon_{1} \epsilon_{4}\left(a_{0}^{+}\right)^{2} \rho_{0}^{+}(1+\zeta)\left\{2 \gamma+(\gamma-1) \zeta+2 b \rho_{0}^{+} \zeta\right\}^{2}\{2 \gamma \\
& +(\gamma-1) \zeta\}^{-2}\left(1-b \rho_{0}^{+}\right)^{-1}+2 U(\gamma+1) \zeta\left\{2 \gamma+(\gamma-1) \zeta-b \rho_{0}^{+}(2 \gamma+(\gamma+1) \zeta)\right\}\{2 \gamma \\
& +(\gamma+1) \zeta\}^{-1}\{2 \gamma+(\gamma-1) \zeta\}^{-1}+2 b\left(a_{0}^{+}\right)^{2} \rho_{0}^{+} U \zeta(1+\zeta)\left\{2 \gamma+(\gamma-1) \zeta+2 b \rho_{0}^{+} \zeta\right\}^{2} \\
& \left.\{2 \gamma+(\gamma+1) \zeta\}^{-1}\{2 \gamma+(\gamma-1) \zeta\}^{-2}\right\}-\frac{\left(a_{0}^{+}\right)^{2} \rho_{0}^{+}(1+\zeta) \nabla \cdot \mathbf{n}}{\gamma U \lambda}\left\{w_{1} d \epsilon_{1} / d \zeta\right. \\
& \left.+w_{2} d \epsilon_{2} / d \zeta-2 \epsilon_{1} \epsilon_{2}+\epsilon_{5}\left\{2 \gamma+(\gamma-1) \zeta+2 b \rho_{0}^{+} \zeta\right\}^{2}\left(\rho_{0}^{+}\right)^{2}\{2 \gamma+(\gamma+1) \zeta\}^{-2}\right\} \\
& \varphi_{4}=-\frac{\left(a_{0}^{+}\right)^{2} \rho_{0}^{+}(1+\zeta)(\nabla \cdot \mathbf{n})^{2}}{\gamma U \lambda}\left\{d \epsilon_{2} / d \zeta+\left(a_{0}^{+}\right)^{2} \rho_{0}^{+}(1+\zeta)\left\{2 \gamma+(\gamma-1) \zeta+2 b \rho_{0}^{+} \zeta\right\}\right. \\
& \{2 \gamma+(\gamma-1) \zeta\}^{-1}+\epsilon^{2}+b \epsilon_{2} \epsilon_{5}\left(a_{0}^{+}\right)^{2} \rho_{0}^{+}(1+\zeta)\left\{2 \gamma+(\gamma-1) \zeta+2 b \rho_{0}^{+} \zeta\right\}^{2}\{2 \gamma \\
& +(\gamma-1) \zeta\}^{-2}\left(1-b \rho_{0}^{+}\right)^{-1}+2 b \zeta\left\{2 \gamma+(\gamma-1) \zeta+2 b \rho_{0}^{+} \zeta\right\}^{2}\{2 \gamma+(\gamma-1) \zeta\}^{-2} \\
& \left.\left(1-b \rho_{0}^{+}\right)^{-1}\right\}+\frac{\epsilon_{3}}{\lambda}\left(a_{0}^{+}\right)^{2} \rho_{0}^{+}(1+\zeta)\left\{2 \gamma+(\gamma-1) \zeta+2 b \rho_{0}^{+} \zeta\right\}\{2 \gamma+(\gamma-1) \zeta\}^{-1} \Delta^{2} \zeta \\
& -\frac{\left(a_{0}^{+}\right)^{2}\left(1-b \rho_{0}^{+}\right) \zeta}{\gamma U} n_{i, j} n_{i, j}+\left\{4 \epsilon_{3}\left(a_{0}^{+}\right)^{2} \rho_{0}^{+}\left(1-b \rho_{0}^{+}\right)(1+\zeta)\{2 \gamma+(\gamma+1) \zeta\}^{-2}\right. \\
& \left.+\epsilon_{3}\right\} \frac{(\nabla \zeta \cdot \nabla \zeta)}{\lambda} .
\end{aligned}
$$

We notice from (7.7) that the behaviour of the coupling term $\Lambda_{(1)}$ depends on another coupling term $\Lambda_{(2)}$, whose determination requires a repetition of the above procedure; proceeding in this manner, we arrive at the following set of transport equations for the coupling terms $\Lambda_{(k)}=\left(n_{i_{1}} n_{i_{2}} \cdots n_{i_{k}} p_{, i_{1} i_{2} \cdots i_{k}}\right)^{-}$for $k=1,2, \cdots$ behind the shock:

$$
\frac{\partial \Lambda_{(k)}}{\partial \tau}+\varphi_{k} \Lambda_{(k+1)}+\Phi_{k}\left(\rho_{0}, p_{0}, \mathbf{n}, \zeta, \Lambda_{(1)}, \Lambda_{(2)}, \cdots, \Lambda_{(k)}\right)=0 ; k=1,2, \cdots
$$

where $\Phi_{k}$ are known functions of their arguments and quantities $\varphi_{k}$ are defined on the shock front.

The infinite set of equations (3.2), (3.3), (3.4), (7.7), and (7.8) with $k=2,3, \cdots$, supplemented by the initial conditions

$$
\mathbf{x}=\mathbf{x}_{0}, \mathbf{n}=\mathbf{n}_{0}, \zeta=\zeta_{0}, \Lambda_{(k)}=\Lambda_{(k)}^{0}, k \geq 1 \text { at } \tau=0,
$$


enable us to determine shock location, orientation, and strength as well as the values of the coupling terms $\Lambda_{(k)}$ at any time $\tau$. It may be noticed that the above infinite system of equations is an open system; in order to provide a natural closure on the infinite hierarchy of equations, we set $\Lambda_{(k+1)}=0$ in (7.8) for the largest $k$ retained [20]. The truncated system, which is closed, can be regarded as a good approximation of the infinite hierarchy of the system governing shock propagation [22]. It is worth mentioning that an infinite sequence of ordinary differential equations, which hold on the shock front, was also derived by Best [31] in an attempt to describe shock motion in one dimension. However, his approach, unlike the one presented here, is based on CCW approximation and admits the fact that the application of a $C_{+}$characteristic equation at the shock is somewhat ad-hoc.

8. Lowest order approximation and the characteristic rule. The lowest order truncation approximation leads to the shock strength equation from (3.1) by setting $(\mathbf{n} \cdot \nabla p)^{-}=0$; this corresponds to the situation where there is no dynamical coupling between the shock and the flow behind the shock. For a three-dimensional configuration, we have $\nabla \cdot \mathbf{n}=m / X$, where $m=0$ corresponds to the planar, $m=1$ corresponds to cylindrically symmetric flows, $m=2$ corresponds to spherically symmetric flows, and $X$ is the radius of the wavefront. Consequently the shock strength equation (3.2) becomes

$$
\frac{d M}{d X}+f_{1}(M) \frac{m}{X}=g_{1}(M) \frac{1}{\rho_{0}^{+}\left(1-b \rho_{0}^{+}\right)} \frac{d \rho_{0}^{+}}{d X},
$$

where $M=U / a_{0}$ is the shock Mach number with

$$
\begin{aligned}
& f_{1}(M)=\frac{\left(M^{2}-1\right)\left\{1+\left(2 M^{2}-1\right) \gamma\right\}\left\{2+(\gamma-1) M^{2}+2 b \rho_{0}^{+}\left(M^{2}-1\right)\right\}}{(\gamma+1) M\left\{4(\gamma+1)+(9 \gamma+1)\left(M^{2}-1\right)+2(2 \gamma-1)\left(M^{2}-1\right)^{2}\right\}}, \\
& g_{1}(M)=\frac{M\left(M^{2}-1\right)\left\{1+\left(2 M^{2}-1\right) \gamma\right\}}{2\left\{4(\gamma+1)+(9 \gamma+1)\left(M^{2}-1\right)+2(2 \gamma-1)\left(M^{2}-1\right)^{2}\right\}} .
\end{aligned}
$$

At this juncture it is worth considering the evolutionary behaviour of the shock using the characteristic rule and comparing the results with the approximation procedure employed here.

The characteristic rule, proposed by Whitham, involves applying the differential relation that is valid along a positive characteristic to the flow quantities immediately behind the shock. The equations in system (2.1) are combined to obtain the following differential equation along positive characteristics:

$$
\frac{D p}{D x}+\rho a \frac{D u}{D x}+\frac{m \rho a^{2} u}{x(u+a)}=0,
$$

where $D / D x=\partial / \partial x+(u+a)^{-1} \partial / \partial t$ denotes the derivative along the characteristic $D x / D t=u+a$. When (8.2) is applied along the shock and jump conditions (2.4) are used, we obtain the following evolutionary equations for the shock:

$$
\frac{D M}{D x}+f_{2}(M) \frac{m}{x}=g_{2}(M) \frac{1}{\rho_{0}^{+}\left(1-b \rho_{0}^{+}\right)} \frac{D \rho_{0}^{+}}{D x},
$$


where

$$
\begin{aligned}
& f_{2}(M)=M\left(M^{2}-1\right)\left\{1+\left(2 M^{2}-1\right) \gamma\right\}\left\{(\gamma+1) M^{2}-2\left(1-b \rho_{0}^{+}\right)\left(M^{2}-1\right)\right\} /\left\{\left\{2 M^{2}\right.\right. \\
& \left.\left\{2+(\gamma-1) M^{2}\right\}^{1 / 2}+\left(M^{2}+1\right)\left\{1+\left(2 M^{2}-1\right) \gamma\right\}^{1 / 2}\right\}\left\{2\left(1-b \rho_{0}^{+}\right)\left(M^{2}-1\right)\{2\right. \\
& \left.\left.\left.\quad+(\gamma-1) M^{2}\right\}^{1 / 2}+\left\{(\gamma+1) M^{2}-2\left(1-b \rho_{0}^{+}\right)\left(M^{2}-1\right)\right\}\left\{1+\left(2 M^{2}-1\right) \gamma\right\}^{1 / 2}\right\}\right\}, \\
& g_{2}(M)=\left\{M\left(M^{2}-1\right)\left(1+\left(2 M^{2}-1\right) \gamma\right)^{1 / 2}\right\} /\left\{4 M^{2}\left(2+(\gamma-1) M^{2}\right)^{1 / 2}\right. \\
& \left.\quad+2\left(M^{2}+1\right)\left(1+\left(2 M^{2}-1\right) \gamma\right)^{1 / 2}\right\} .
\end{aligned}
$$

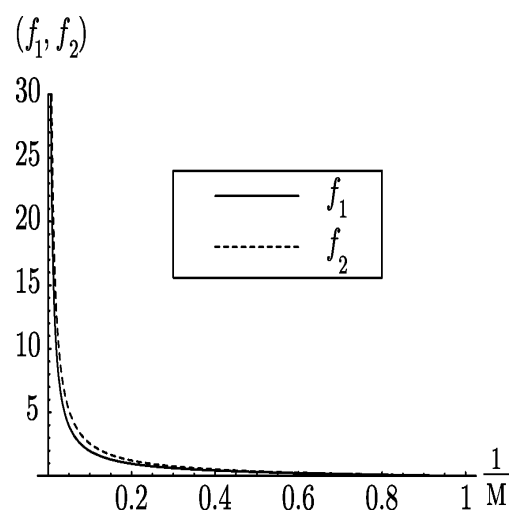

FIG. 1. Functions $f_{1}(M)$ and $f_{2}(M)$, which appear in the characteristic rule and the lowest order truncation approximation versus $1 / M ;$ here $\gamma=1.4$

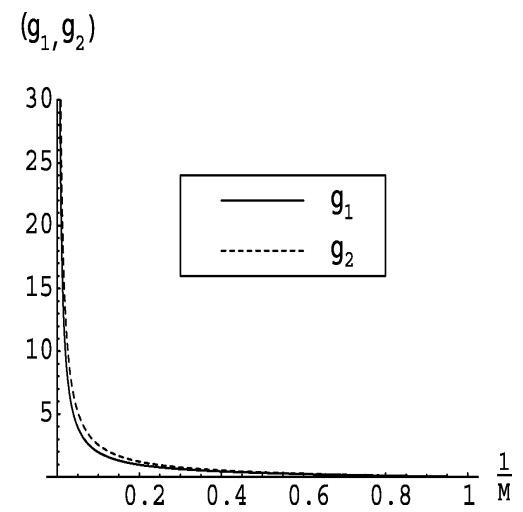

FIG. 2. Functions $g_{1}(M)$ and $g_{2}(M)$, which appear in the characteristic rule and the lowest order truncation approximation versus $1 / M ;$ here $\gamma=1.4$

It is interesting to notice that equation (8.1), obtained by using the lowest order approximation, closely resembles equation (8.3) obtained from the characteristic rule. Indeed, it is remarkable that, in the case of weak shock, the functions $f_{1}(M)$ and $f_{2}(M)$ and $g_{1}(M)$ and $g_{2}(M)$ exhibit the same asymptotic behaviour, namely that both $f_{1}$ and 
$f_{2} \rightarrow \frac{1}{2}(M-1)$ and $g_{1}$ and $g_{2} \rightarrow \frac{1}{4}(M-1)$ as $M \rightarrow 1$; however, for strong shocks, the lowest order truncation rule gives the following form of the functions $f_{1}(M)$ and $g_{1}(M)$ and $f_{2}(M)$ and $g_{2}(M)$ as $M \rightarrow \infty$ :

$$
\begin{aligned}
f_{1}(M) & \sim \frac{(M-1) \gamma\left(\gamma-1+2 b \rho_{0}^{+}\right)}{(2 \gamma-1)(\gamma+1)} \\
g_{1}(M) & \sim \frac{M \gamma}{2(2 \gamma-1)} \\
f_{2}(M) & \sim \frac{(M-1) 2 \gamma\left(\gamma-1+2 b \rho_{0}^{+}\right)}{\left\{2(\gamma-1)^{1 / 2}+(2 \gamma)^{1 / 2}\right\}\left\{2\left(1-b \rho_{0}^{+}\right)(\gamma-1)^{1 / 2}+\left(\gamma-1+2 b \rho_{0}^{+}\right)(2 \gamma)^{1 / 2}\right\}} \\
g_{2}(M) & \sim \frac{M(2 \gamma)^{1 / 2}}{2\left\{(2 \gamma)^{1 / 2}+2(\gamma-1)^{1 / 2}\right\}}
\end{aligned}
$$

For the sake of comparison of $f_{1}(M)$ and $f_{2}(M)$ and $g_{1}(M)$ and $g_{2}(M)$ throughout the range $1 \leq M<\infty$, Fig. 1 and Fig. 2 show their variation as a function of $M^{-1}$; inspection of the curves makes it clear that the evolutionary behaviour of shocks described by the lowest order approximation is close to that predicted by the characteristic rule.

8.1. Special Cases. Case $\mathbf{I}$ : Let us consider $\rho_{0}^{+}=$Const., i.e., the shock is propagating into a uniform state with constant density, then (8.1) under strong shock limit $(M>>1)$ yields

$$
M=M_{0}\left(X / X_{0}\right)^{-m \beta_{1}},
$$

where $\beta_{1}=\left\{\gamma\left(\gamma-1+2 b \rho_{0}^{+}\right)\right\} /\{(2 \gamma-1)(\gamma+1)\}$,

while the CCW approximation gives

$$
M=M_{0}\left(X / X_{0}\right)^{-m \beta_{2}},
$$

where $\beta_{2}=\left\{2 \gamma\left(\gamma-1+2 b \rho_{0}^{+}\right)\right\} /\left\{\left\{2(\gamma-1)^{1 / 2}(2 \gamma)^{1 / 2}\right\}\left\{2\left(1-b \rho_{0}^{+}\right)(\gamma-1)^{1 / 2}+(\gamma-1+\right.\right.$ $\left.\left.\left.2 b \rho_{0}^{+}\right)(2 \gamma)^{1 / 2}\right\}\right\}$.

Table 1 shows a comparison between the exponents $\beta_{1}$ and $\beta_{2}$ within the context of van der Waals' excluded volume $b$; it may be remarked that the results are very close to each other.

TABLE 1. The asymptotic behaviour of the exponents $\beta_{1}$ and $\beta_{2}$

\begin{tabular}{|c|c|c|c|c|}
\hline$b \rho_{0}^{+}$ & \multicolumn{2}{|c|}{ Whitham rule $\left(\beta_{2}\right)$} & \multicolumn{2}{c|}{ Present Case $\left(\beta_{1}\right)$} \\
\hline & $\gamma=1.4$ & $\gamma=1.6$ & $\gamma=1.4$ & $\gamma=1.6$ \\
\hline 0.4 & 0.41 & 0.39 & 0.39 & 0.39 \\
\hline 0.42 & 0.42 & 0.38 & 0.40 & 0.40 \\
\hline 0.44 & 0.43 & 0.40 & 0.42 & 0.41 \\
\hline 0.46 & 0.44 & 0.41 & 0.43 & 0.42 \\
\hline 0.48 & 0.44 & 0.42 & 0.44 & 0.44 \\
\hline 0.50 & 0.45 & 0.42 & 0.45 & 0.45 \\
\hline
\end{tabular}


Case II : Let us consider the specific dependence of $\rho_{0}^{+}$on $x$ given by the relation (see Whitham [9])

$$
\rho_{0}^{+}(x)=\rho_{c} e^{-\left(x / x_{0}\right)},
$$

where $\rho_{c}$ and $x_{0}$ are some reference constants. Then equation (8.1) under the weak shock limit yields

$$
M=1+k_{1}\left(M_{0}-1\right) x^{-m / 2}\left(e^{x}-b \rho_{c}\right)^{-1 / 4},
$$

where $x \equiv x / x_{0}$ and $k_{1}=\left(e-b \rho_{c}\right)^{1 / 4}$. Equation (8.4) shows that the dissipative mechanism on account of the presence of van der Waals' excluded volume $b$ causes a weak shock to attenuate faster as compared to what it would be in an ideal gas case $(b=0)$; the corresponding situation is illustrated by the curves in Fig. 3 . Furthermore, it is evidently clear from (8.4) that a spherical shock decays faster than a cylindrical wave or a plane wave as one would expect (see Fig. 4).

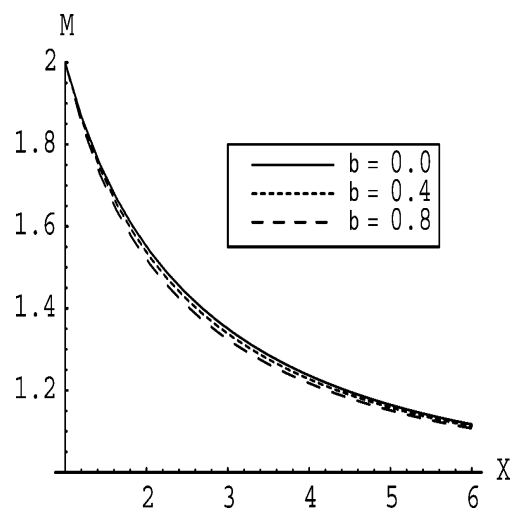

Fig. 3. The behaviour of $M$ influenced by the van der Waals excluded volume $b$, for cylindrical symmetric flows.

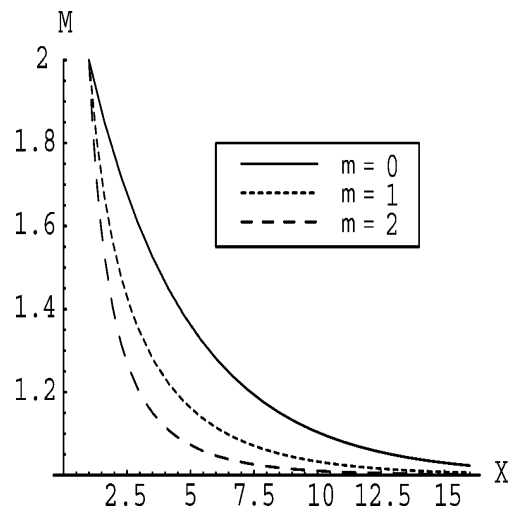

FIG. 4. The decay behaviour of $M$ for Planar $(m=0)$, Cylindrical $(m=1)$ and Spherical $(m=2)$ waves, here $b=0.2$ 
9. Conclusion. This article describes an approximate analytical method for determining the evolutionary behaviour of a three-dimensional shock of arbitrary strength in a non-ideal gas; the work derives motivation from the study relating to an intrinsic description of shock wave propagation carried out in the past. A rigorous mathematical approach is used to derive an infinite system of transport equations that hold on the shock front; this infinite system allows investigation of the dynamical coupling that exists between the shock and the rearward flow. The solutions of the truncated finite system efficiently describe the shock motion; recently, Batt and Ravindran [27] have provided a rigorous justification of this approximation within the context of the inviscid Burgers equation, which has certain features in common with the system appearing in this paper, where we use the singular surface theory to represent the shock as a propagating singular surface and study its evolutionary behaviour by considering a sequence of transport equations for the variation of jumps in pressure and its space derivatives across the shock. Our basic transport equations for a two-dimensional shock motion, which bear a close structural resemblance with those of geometrical shock dynamics, enable us to describe the onset of shock-shock and determine the effects of van der Waals' excluded volume and the wavefront geometry on the evolutionary behaviour of shock waves. The evolutionary behaviour of weak and strong shocks described by the lowest order truncation approximation is found to be in excellent agreement with the results predicted by the CCW approximation; indeed the two evolutionary laws exactly coincide in the weak shock limit. The dissipative mechanism on account of the presence of van der Waals' excluded volume $b$ causes a weak shock to attenuate faster as compared to what it would be in an ideal gas case $(b=0)$; the corresponding situation is illustrated by the curves in Fig. 3. Furthermore, it is evidently clear from (8.4) that a spherical shock decays faster than a cylindrical wave or a plane wave as one would expect (see Fig. 4). For imploding shocks, the value of the exponent for a spherical or cylindrical case is compared with that obtained using CCW approximation; it may be remarked that an infinite sequence of ordinary differential equations, which hold on the shock front, was also derived by Best 31 in an attempt to describe shock motion in one dimension. However, his approach, unlike the one presented here, is based on CCW approximation and admits the fact that the application of a $C_{+}$characteristic equation at the shock is somewhat ad-hoc. We conclude this section with a remark regarding the mathematical structure noted in Section 3, where the governing Euler system of equations is used to derive equations for the jump in flow quantities. Indeed, implicit in this development is the assumption that the flow on both sides of the shock is smooth. The case in which the flow behind a shock,

near the triple point, is not smooth yields many technical difficulties, and we postpone it to a future work.

\section{REFERENCES}

[1] Courant, R. and Friedrichs, K. O., Supersonic flow and shock waves, Springer, New York (1999). MR 0421279 (54:9284)

[2] Lighthill, M. J., Higher approximations in aerodynamic theory, Princeton University Press, Princeton, N.J., (1960).

[3] Meyer, R. E., In Handbuch der Physik, Vol. IX, edited by S. Flugge (Springer-Verlag, Berlin) (1960). MR0119653(22:10414) 
[4] Ardavan - Rhad, H., The decay of a plane shock wave, J. Fluid Mech. 43 (1970), 737-751.

[5] Sirovich, L. and Chong, T. H., Approximate solution in gasdynamics, Phys. Fluids 23 (1980), 1291-1295. MR578116 (81d:76071)

[6] Sharma, V. D., Ram, R. and Sachdev, P., Uniformly valid analytical solution to the problem of decaying shock wave, J. Fluid Mech. 185 (1987) 153-170.

[7] Chong, T. H. and Sirovich, L., Numerical integration of the gasdynamic equation, Phys. Fluids 23 (1980), 1296-1300.

[8] Lewis, T. S. and Sirovich, L., Approximate and exact numerical supersonic flow over an airofoil, J. Fluid Mech. 112 (1981), 265-282.

[9] Whitham, G. B., Linear and nonlinear waves, Wiley, New York (1974). MR0483954 (58:3905)

[10] Collins, R. and Chen, H. T., Propagation of shock wave of arbitrary strength in two half planes containing a free surface, J. Comp. Phys. 5 (1970), 415-422.

[11] Collins, R. and Chen, H. T., Motion of a shock wave through a non-uniform fluid, In Proc. 2nd Int. Conf. on Numerical Methods in Fluid Dynamics (ed. M. Holt). Lecture Notes in Physics. Vol. 8, pp. 264-269. Springer.

[12] Catherasoo, C. J. and Sturtevant, B., Shock dynamics in nonuniform media, J. Fluid Mech. 127, (1983) 539-561. MR698526 (84e:76044)

[13] Henshaw, W. D., Smyth, N. F. and Schwendeman, D. W., Numerical shock propagation using geometrical shock dynamics, J. Fluid Mech. 171 (1986), 519-545.

[14] Schwendeman, D. W., A numerical scheme for shock propagation in three dimensions, Proc. R. Soc. London Ser. A 416 (1988), 179-198. MR936120 (89c:76077)

[15] Cates, J. E. and Sturtevant. B., Shock wave focusing using geometrical shock dynamics, Phys. Fluids 9 (1997), 3058-3068. MR.1472445

[16] Schwendeman, D. W., A new numerical method for shock wave propagation on geometrical shock dynamics, Proc. R. Soc. London, A 441 (1993), 331-341. MR1219259(94i:76046)

[17] Nunziato, J. W. and Walsh, E. K., Propagation and growth of shock waves in inhomogeneous fluids, Phys. Fluids 15 (1972), 1397-1402.

[18] Chen, P. J., Selected topics in wave propagation, Noordhoff, Leyden (1976).

[19] Wright, T. W., An intrinsic description of unsteady shock waves, Q. J. Mech. Appl. Math. 29 (1976), 311-324.

[20] Maslov, V. P., Propagation of shock waves in an isentropic non viscous gas, J. Soviet Math. 13 (1980), 119-163.

[21] Grinfeld, M. A., Ray method of calculating the wavefront intensity in nonlinearly elastic material, PMM 42 (1978), 958-977. MR620884 (82j:73024)

[22] Anile, A. M. and Russo, G., Generalized wavefront expansion I : Higher order corrections for the propagation of weak shock waves, Wave Motion 8 (1986), 243-258. MR841962 (87h:76081)

[23] Fu, Y. B. and Scott, N. H., One dimensional shock waves in simple materials with memory, Proc. R. Soc. Lond. A 428 (1990), 547-571. MR1050540 (91k:73048)

[24] Sharma, V. D. and Radha, Ch., On one-dimensional planar and non-planar shock in a relaxing gas, Phys. Fluids 6 (1994), 2177-2190. MR1275083 (95b:76053)

[25] Ravindran, Renuka, Strong shock approximation in the new theory of shock dynamics, Quart. J. Mech. Appl. Math. 50 (1997), 251-259. MR1451070(98d:76101)

[26] Radha, Ch., Sharma, V. D., and Jeffrey, A., An approximate analytical method for describing the kinematics of a bore over a sloping beach, Appl. Anal. 81 (2002), 867-892. MR 1929550|(2003h:76054)

[27] Batt, Jürgen and Ravindran, Renuka, Calculation of shock using solutions of systems of ordinary differential equations, Quart. Appl. Math. 63 (2005), 721-746. MR2187929 (2007f:35245)

[28] Truesdell, C. and Toupin, R. A., The classical field theories, In Handbuch der Physik, Vol. III, Berlin, Springer-Verlag (1960). MR0118005 (22:8778)

[29] Menon, V. V, Sharma, V. D., and Jeffrey, A., On the general behavior of acceleration waves, Applicable Analysis 16 (1983), 101-120. MR709814 (84h:73014)

[30] Sharma, V. D. and Menon, V. V., Further comments on the behavior of acceleration waves of arbitrary shape, J. Math. Phys. 22 (1981), 683-684.

[31] Best, J. P., A generalization of the theory of geometrical shock dynamics, Shock Waves, 1 (1991), 251-273. 